Research Paper

\title{
The Value of LncRNA BCAR4 as a Prognostic Biomarker on Clinical Outcomes in Human Cancers
}

\author{
Chao $\mathrm{Tu}^{1,2,4^{*}}$, Xiaolei Ren ${ }^{1,2^{*}}$, Jieyu He ${ }^{3}$, Chenghao Zhang ${ }^{1,2}$, Ruiqi Chen ${ }^{1,2}$, Wanchun Wang ${ }^{1,2}$, Zhihong \\ $\mathrm{Li}^{1,2}$ \\ 1. Department of Orthopedics, The Second Xiangya Hospital, Central South University, Changsha, Hunan, P.R. China. \\ 2. Hunan Key Laboratory of Tumor Models and Individualized Medicine, The Second Xiangya Hospital, Central South University, Changsha, Hunan, P.R. \\ China. \\ 3. Department of Geriatrics, The Second Xiangya Hospital, Central South University, Changsha, Hunan, P.R. China. \\ 4. University of Texas Health Science Center at San Antonio, San Antonio, Texas, USA. \\ *These authors contributed equally. \\ $\triangle$ Corresponding author: Zhihong Li, M.D, Ph.D. Department of Orthopedics, the Second Xiangya Hospital, Central South University, Changsha 410011, \\ Hunan, China; Tel: +86 0731-85295127; Fax: +86 0731-85294041; Email: lizhihong@csu.edu.cn \\ (c) The author(s). This is an open access article distributed under the terms of the Creative Commons Attribution License (https://creativecommons.org/licenses/by/4.0/). \\ See http://ivyspring.com/terms for full terms and conditions.
}

Received: 2019.03.21; Accepted: 2019.08.25; Published: 2019.10.15

\begin{abstract}
Background: This updated meta-analysis aimed to analyze available data to explore the prognostic value of long noncoding RNA breast cancer anti-estrogen resistance 4 (BCAR4) in various human malignancies. Methods: Literature retrieval was performed by systematic searching several authoritative databases, including Pubmed, PMC database, Web of Science, the Cochrane Library, Embase, and CNKI database up to Feb 10, 2019. Data were extracted and subsequently crosschecked, and discrepancies were discussed to reach consensus. Quality of the eligible studies was evaluated by Newcastle-Ottawa Scale (NOS). The fixed- or random-effects model was used to calculate the pooled the hazard ratios (HRs) or odds ratios (ORs) and the $95 \%$ confidence interval $(95 \% \mathrm{Cl})$. Publication bias was detected by using Begg's funnel plot and Egger's test. Results: A total 1,128 cancer patients from thirteen studies were included and pooled in the present meta-analysis. High expression levels of BCAR4 were correlated with unfavorable overall survival (OS) (HR=2.23, $95 \% \mathrm{Cl}$ : 1.84-2.71), but not progression-free survival (PFS) (HR=1.30, $95 \% \mathrm{Cl}: 0.80-2.11$ ). Subgroup stratified analysis showed that tumor type, sample size, follow-up months, and survival analysis method did not alter the predictive value of BCAR4 on OS in various cancers. Furthermore, elevated BCAR4 level was markedly correlated with advanced clinical stage (III/IV) $(O R=3.28,95 \%$ $\mathrm{Cl}$ : 2.33-4.60), and dramatically predicted lymph node metastasis (OR=3.00, 95\% Cl: 1.95-4.63, $P<0.00001)$ and distant metastasis ( $O R=3.36,95 \% \mathrm{Cl}: 1.88-5.98, P<0.0001)$, but not associated with age, gender or tumor size. No obvious heterogeneity was noted for correlation between BCAR4 expression and OS across these studies. Conclusions: High expression of BCAR4 was correlated with unfavorable overall survival outcome and clinical features including metastasis and progression, implicating an independent prognostic value for BCAR4 in human cancers.
\end{abstract}

Key words: LncRNA; BCAR4; cancer, sarcoma; prognosis; metastasis

\section{Introduction}

Cancer is predicted to rank as the leading cause of mortality and the single most critical barrier to increase life expectancy worldwide over the past decades [1]. An estimated 18.1 million new cancer cases and 9.6 million cancer deaths worldwide in 2018 were reported by the International Agency for Research on Cancer [1]. Despite tremendous achievements have been made in surgery, adjuvant radio- and chemotherapy, targeted therapy [2], and even immunotherapy [3] in the past decades, the 
prognosis and quality of life of cancer patients still remain poor, largely due to the shortage of effective and noninvasive predictive factors during early stage of malignancies. Therefore, many researchers have been devoted to exploration of new promising putative biomarkers for prognosis and therapeutic efficacy for cancer patients, and finally improve their survival outcomes [4].

Non-coding RNAs (ncRNAs) refer to a variety type of RNA with a profound role in epigenetic regulation at the transcriptional and posttranscriptional level, including hetero-chromatin formation, DNA methylation, gene silencing, and histone modification [5-7]. Generally, the epigenetic related ncRNAs can be simply separated into two main categories by their size: those with nucleotides less than $30 \mathrm{nts}$ are short ncRNAs, such as microRNAs (miRNAs), piwi-interacting RNAs (piRNAs), and short interfering RNAs (siRNAs), while those longer than $200 \mathrm{nts}$ belong to long ncRNAs (lncRNAs) [8-10].

LncRNAs could drive many pathophysiologic phenotypes through their interaction with other cellular macro-molecules including DNA, RNA and proteins [11]. Accumulating evidences have linked expression or functional abnormalities of lncRNA with various complex human disease, such as aging [12], degenerative disease [13], and coronary artery disease [14]. Recently, lncRNAs have been reported to function in biological processes associated with cancer initiation and progress including proliferation, apoptosis and invasion, and therefore implicate a putative role in tumorigenesis [7, 15-17].

Breast cancer anti-estrogen resistance 4 (BCAR4) gene produces a spliced lncRNA that has been firstly identified to be inversely associated with the development of resistance to anti-estrogens in breast cancer cells and poor disease-free survival (DFS) for recurrent breast cancer. Previously, lncRNA BCAR4 was considered as an oncogene and was reported to play a pivotal role in metastasis and tamoxifenresistance of breast cancer [18]. Recently, many researches showed that the dramatically elevated expression pattern of BCAR4 was closely correlated with worse survival and high risk of metastasis in other cancer patients as well [19]. The expression of BCAR4 was higher in various tumor tissues than normal tissue or para-tumor tissue, including breast cancer [20-22], non-small-cell lung cancer [23-25], prostate cancer [26], osteosarcoma [27, 28], gastric cancer [29], cervical cancer [30] and colorectal cancer $[31,32]$. However, most individual studies evaluating BCAR4 expression in cancers remain insufficient due to the limitations in small sample size and possible controversial outcomes. Accordingly, we conducted this comprehensive meta-analysis with all related eligible studies and pooled results to further address the feasibility of BCAR4 as a noninvasive prognostic biomarker candidate.

\section{Material and methods}

\section{Search strategy and literature selection}

Potential eligible literature that related to the prognosis and metastasis of BCAR4 and human cancer were thoroughly searched in related databases, including Pubmed, PMC database, Web of Science, the Cochrane Library and Embase, as well as Chinese databases: China National Knowledge Infrastructure (CNKI) from inception to Feb 10, 2019. The searched terms in variably combinations were listed as follows: ("long noncoding RNA-, lncRNA-, breast cancer anti-estrogen resistance 4, BCAR4,") and ("carcinoma" or "sarcoma" or "cancer" or "tumor" or "tumour" or "neoplasm" or "malignancy"). An additional manual search of references lists of primary literature was performed to find supplementary pertinent articles. Notably, the current study was critically projected, reviewed and reported in accordance with the PRISMA checklist to enhance the credibility of the results $[33,34]$.

\section{Inclusion and exclusion criteria}

Rigorous inclusion and exclusion criteria were adopted in this study. Inclusion criteria were as following: 1) articles examining the clinical prognostic value of BCAR4 in any malignancies; 2) the patients had been grouped according to the BCAR4 expression (high versus low); 3) definite diagnosis with histopathology confirmation; 4) Adequate data for the measurement of hazard ratios (HRs) or odds ratios (ORs) and the corresponding 95\% confidence intervals (CIs); and 5) published in English or Chinese language.

By contrast, exclusion criteria were as following: 1) literature not pertinent to the BCAR4;2) studies concerning the structure or functions of BCAR4; 3) multiple duplicate publications or duplicate data in the different works, excluding smaller sample data; 4) animal experiments, and 5) absence of usable clinical data or documents without original data, such as correspondences, editorial materials, case reports or reviews.

\section{Data extraction and quality assessment}

Two investigators (CT and XLR) extracted all the essential information from identical articles independently, and a third investigator (JYH) was consulted to reach a consensus when inconsistencies exist between the investigators. Extracted information are as following: 1) first author' name, year of publication, origin country, tumor type, sample size, 
follow-up months, cut-off value, clinical TNM stage, detection and survival analysis method; 2) HRs or ORs with 95\% CI of BCAR4 for overall survival (OS), progression-free survival (PFS), DFS, recurrence-free survival (RFS), lymph node metastasis (LNM) and distant metastasis (DM). If only Kaplan-Meier curves were provided in certain studies, the survival rates were indirectly extracted from the graphical plots and calculated HRs with 95\% CIs were determined by using Engauge Digitizer software (Version 4.1) as previously described [35]. Moreover, best efforts were made by contacting the corresponding author to obtaining the possible data if they were not available from the enrolled articles.

Quality assessment of the eligible literature was performed by two independent investigators ( $\mathrm{CHZ}$ and RQC) through using the Newcastle-Ottawa Scale (NOS), and the studies with NOS score $\geq 7$ were considered to be of high quality [36].

\section{Data synthesis and statistical analysis}

All statistical analyses were conducted on STATA software (Version 12.0) and Review Manager (RevMan 5.3). The impact of BCAR4 expression on clinical characteristics, prognosis and metastasis was described as HRs or ORs with corresponding 95\% CIs. Heterogeneity among the included studies was quantified with the by chi-squared test and $I^{2}$ statistics. A chi-squared test of $p<0.10, \quad I^{2}>50 \%$ indicated significant heterogeneity across the studies, and the random-effects model should be adopted in analyzing the pooled results. On the contrary, the fixed-effects model could be applied in data analysis when no obvious heterogeneity was detected (chi-squared test of $p>0.10$ and $I^{2}<50 \%$ ). Probable publication bias was estimated by employing Begg's funnel plot as well as Egger's regression test. $P<0.05$ of the two-tailed probability was considered to be statistically significant.

\section{Verification of results from TCGA and GTEx dataset}

Gene Expression Profiling Interactive Analysis (GEPIA) was additionally used in this meta-analysis in order to further verify the expression levels of BCAR4 in cancerous and normal tissue and its correlations with OS and DFS from The Cancer Genome Atlas (TCGA) and GTEx dataset. The survival analysis was calculated by Kaplan-Meier (K-M) method and logrank test, and the HRs and $\mathrm{p}$-value were shown in the figure of K-M curves as previously described [37].

\section{Results}

\section{Included literature}

A total of 284 references were retrieved through initial searches of the electronic databases above-mentioned. 77 duplication articles were excluded firstly after screening. 164 articles, including 110 studies on irrelevant topics and 54 reviews or meeting abstracts, were further excluded according to the inclusion and exclusion criteria after examination by title and abstract. For the remaining 43 potential candidate studies, full texts were further carefully reviewed, and 15 articles were excluded as survival analysis was not described, nine studies were duplicate reports from the same research organizations, and six are abstracts which data are not extractable. Ultimately, thirteen articles were included and used in quantitative synthesis for the present systematic review and meta-analysis. The selection process was briefly presented in the flow diagram in Figure 1.

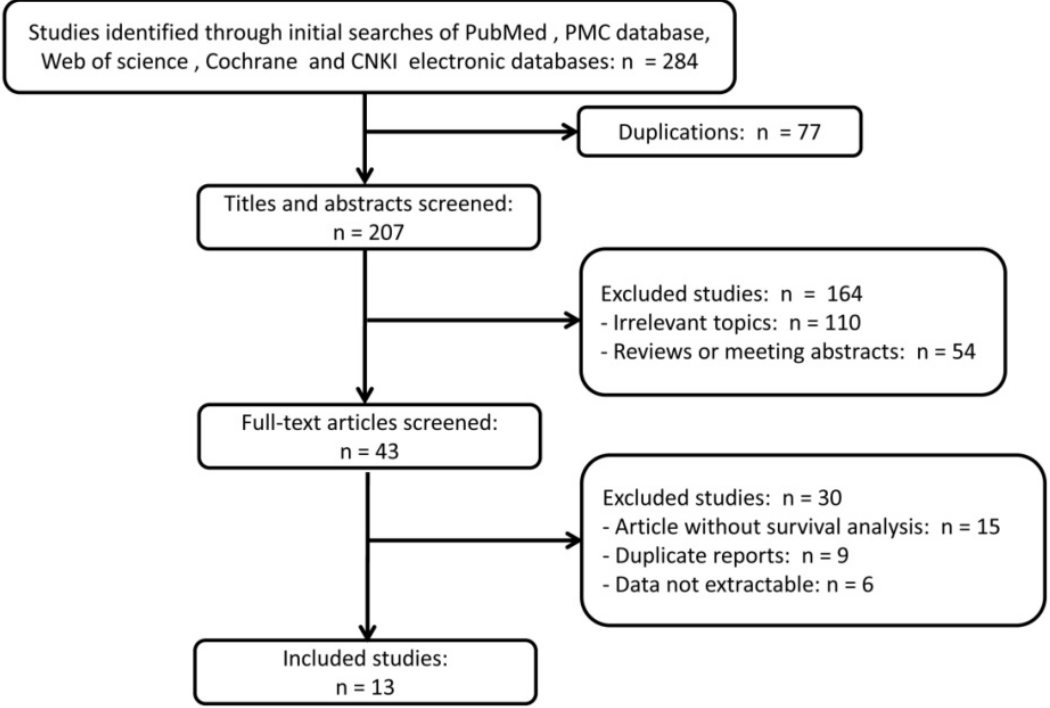

Figure 1. Flow diagram of study identification with criteria in the meta-analysis. 


\section{Characteristics of the enrolled studies}

The main features of the thirteen included articles are concisely summarized in Table 1 . These studies were published between 2010 and 2018 with sample size ranging from 30 to 168 . The median or mean value was selected as the cut-off value in most articles. Eleven of the studies were obtained from China, whereas one from the US and another from the Netherlands. Out of the thirteen studies, three emphasized breast cancer, while another was based on castration-resistant prostate cancer. Additionally, two of them focused on osteosarcoma, with another three focusing on non-small cell lung cancer. There were also two based on colorectal cancer, another one taking into gastric cancer, and the last one emphasized cervical cancer. All of the 1,128 patients were divided into two distinct groups (high and low expression of BCAR4) as measured by qRT-PCR or RNA in situ hybridization. Ten of thirteen studies investigated the association between BCAR4 expression and OS, while only two depicted BCAR4's prognostic role in PFS.

The NOS score indicated the overall good quality of the studies (median, 7.8 points; range, 7-9), with no manuscripts displaying high risk of bias.

\section{BCAR4 and main survival outcome}

The fixed-effects model was used to analyze the pooled HRs and corresponding 95\% CIs since no obvious heterogeneity was noted among the studies involved in OS and PFS analysis (chi-squared test of $p>0.10$ and $I^{2}<50 \%$ ). Elevated BCAR4 expression was predictive of unfavorable OS $(\mathrm{HR}=2.23,95 \% \mathrm{CI}$ : 1.84-2.71), but not PFS (HR=1.30, 95\% CI: 0.80-2.11) in various carcinomas according to the results (Figure 2).
Afterwards the stratified analyses were conducted by tumor type, sample size (more or less than 100), follow-up months (more or less than 60), and survival analysis method to further analyze the BCAR4 expression with OS, as displayed in Table 2. The results showed that all these subgroup analysis parameters did not alter the prognostic value of BCAR4 on OS (Figure 3). Of note, for studies assessing OS in different tumor types, the results indicated that promoted BCAR4 levels could significantly predict worse outcome in breast cancer, gastrointestinal cancer, and osteosarcoma with pooled HRs with 95\% CIs of $2.44(1.22,4.85), 2.04(1.55,2.69)$ and 2.58 (1.38, 4.80) respectively as shown in Figure 3.

\section{BCAR4 and other clinicopathological features}

The characteristics of the enrolled studies which assessing the correlation between BCAR4 expression and other clinicopathological features including metastasis were summarized in Table 3.

The results showed that elevated BCAR4 expression was positively associated with advanced clinical TNM stage (III/IV vs. I/II) with estimated OR and $95 \%$ CI of $3.28(2.33,4.60)$, while age, gender and tumor size showed no correlation with BCAR4 level (Figure 4).

In addition, the pooled ORs have revealed that BCAR4 expression might be regarded as an independent prognostic biomarker for aggressiveness and metastasis in human cancers. As presented in Figure 5, promoted BCAR4 expression strongly predicted LNM (OR=3.00, 95\% CI: 1.95-4.63, $P<0.00001)$ and DM (OR=3.36, 95\% CI: 1.88-5.98, $P<0.0001)$ respectively.

Table 1. Summary of the main characteristics of the included studies.

\begin{tabular}{|c|c|c|c|c|c|c|c|c|c|c|c|c|c|}
\hline \multirow[t]{2}{*}{ First Author } & \multirow[t]{2}{*}{ Year } & \multirow[t]{2}{*}{ Country } & \multirow[t]{2}{*}{ Tumor Type } & \multirow[t]{2}{*}{$\begin{array}{l}\text { TNM } \\
\text { Stage }\end{array}$} & \multirow[t]{2}{*}{$\begin{array}{l}\text { Sample } \\
\text { Size }\end{array}$} & \multicolumn{2}{|c|}{$\begin{array}{l}\text { BCAR4 } \\
\text { expression }\end{array}$} & \multirow[t]{2}{*}{$\begin{array}{l}\text { Cutoff } \\
\text { Value }\end{array}$} & \multirow[t]{2}{*}{$\begin{array}{l}\text { Follow-up } \\
\text { (months) }\end{array}$} & \multirow[t]{2}{*}{$\begin{array}{l}\text { Detection } \\
\text { Method }\end{array}$} & \multirow[t]{2}{*}{$\begin{array}{l}\text { Survival } \\
\text { Analysis }\end{array}$} & \multirow[t]{2}{*}{$\begin{array}{l}\text { Outcome } \\
\text { Measure }\end{array}$} & \multirow[t]{2}{*}{ NOS } \\
\hline & & & & & & High & Low & & & & & & \\
\hline Cai, Z [26] & 2018 & China & PC & II-IV & 40 & $\mathrm{~N} / \mathrm{A}$ & $\mathrm{N} / \mathrm{A}$ & Median & 60 & qRT-PCR & Univariate & OS & 7 \\
\hline Chen, F [27] & 2016 & China & Osteosarcoma & I-IV & 60 & 30 & 30 & Median & 60 & qRT-PCR & Multivariate & OS/RFS & 7 \\
\hline Gong, J [23] & 2017 & China & NSCLC & I-IV & 68 & 35 & 33 & Mean & 60 & qRT-PCR & Multivariate & OS & 8 \\
\hline $\mathrm{Ju}, \mathrm{L}[28]$ & 2016 & China & Osteosarcoma & IIA-III & 168 & 87 & 81 & $\mathrm{~N} / \mathrm{A}$ & 70 & qRT-PCR & Multivariate & OS & 8 \\
\hline $\begin{array}{l}\text { L. WANG } \\
{[29]}\end{array}$ & 2017 & China & GC & $\mathrm{N} / \mathrm{A}$ & 113 & $\mathrm{~N} / \mathrm{A}$ & $\mathrm{N} / \mathrm{A}$ & Mean & 150 & qRT-PCR & Multivariate & OS & 9 \\
\hline $\mathrm{Li}, \mathrm{Q}[31]$ & 2016 & China & CRC & I-IV & 30 & 15 & 15 & $\mathrm{~N} / \mathrm{A}$ & 30 & qRT-PCR & Univariate & OS & 7 \\
\hline $\begin{array}{l}\text { MFE } \\
\text { Godinho [20] }\end{array}$ & 2010 & $\begin{array}{l}\text { The } \\
\text { Netherlands }\end{array}$ & $\mathrm{BC}$ & $\mathrm{N} / \mathrm{A}$ & 81 & 40 & 41 & Median & 120 & qRT-PCR & Univariate & PFS/MFS & 9 \\
\hline N. Li [25] & 2017 & China & NSCLC & I-IV & 76 & 38 & 38 & $\mathrm{~N} / \mathrm{A}$ & $\mathrm{N} / \mathrm{A}$ & qRT-PCR & $\mathrm{N} / \mathrm{A}$ & None & 7 \\
\hline $\begin{array}{l}\text { Ouyang, S } \\
{[32]}\end{array}$ & 2017 & China & CRC & I-III & 60 & $\mathrm{~N} / \mathrm{A}$ & $\mathrm{N} / \mathrm{A}$ & $\mathrm{N} / \mathrm{A}$ & 90 & $\begin{array}{l}\text { RNA } \\
\text { Hybridization }\end{array}$ & Univariate & OS/DFS & 8 \\
\hline Xing, Z [21] & 2014 & The US & $\mathrm{BC}$ & $\mathrm{N} / \mathrm{A}$ & 160 & $\mathrm{~N} / \mathrm{A}$ & $\mathrm{N} / \mathrm{A}$ & $\mathrm{N} / \mathrm{A}$ & 150 & qRT-PCR & Univariate & OS & 7 \\
\hline Yang, H [24] & 2018 & China & NSCLC & $\mathrm{N} / \mathrm{A}$ & 64 & 51 & 13 & $\mathrm{~N} / \mathrm{A}$ & 64 & qRT-PCR & Univariate & None & 7 \\
\hline $\begin{array}{l}\text { Zhang, JB } \\
\text { [22] }\end{array}$ & 2017 & China & $\mathrm{BC}$ & I-IV & 80 & 47 & 33 & N/A & $<60$ & qRT-PCR & Univariate & OS & 8 \\
\hline Zou, R [30] & 2018 & China & $\begin{array}{l}\text { Cervical } \\
\text { cancer }\end{array}$ & I-IV & 128 & 64 & 64 & Median & 60 & qRT-PCR & Multivariate & OS/PFS & 9 \\
\hline
\end{tabular}

Abbreviations: BC, breast cancer; CRC, colorectal cancer; DFS, disease-free survival; GC, gastric cancer; MFS, metastasis-free survival; N/A, not available; NSCLC, non-small cell lung cancer; OS, overall survival; PC, Prostate cancer; PFS, progression-free survival; RFS, recurrence-free survival. 


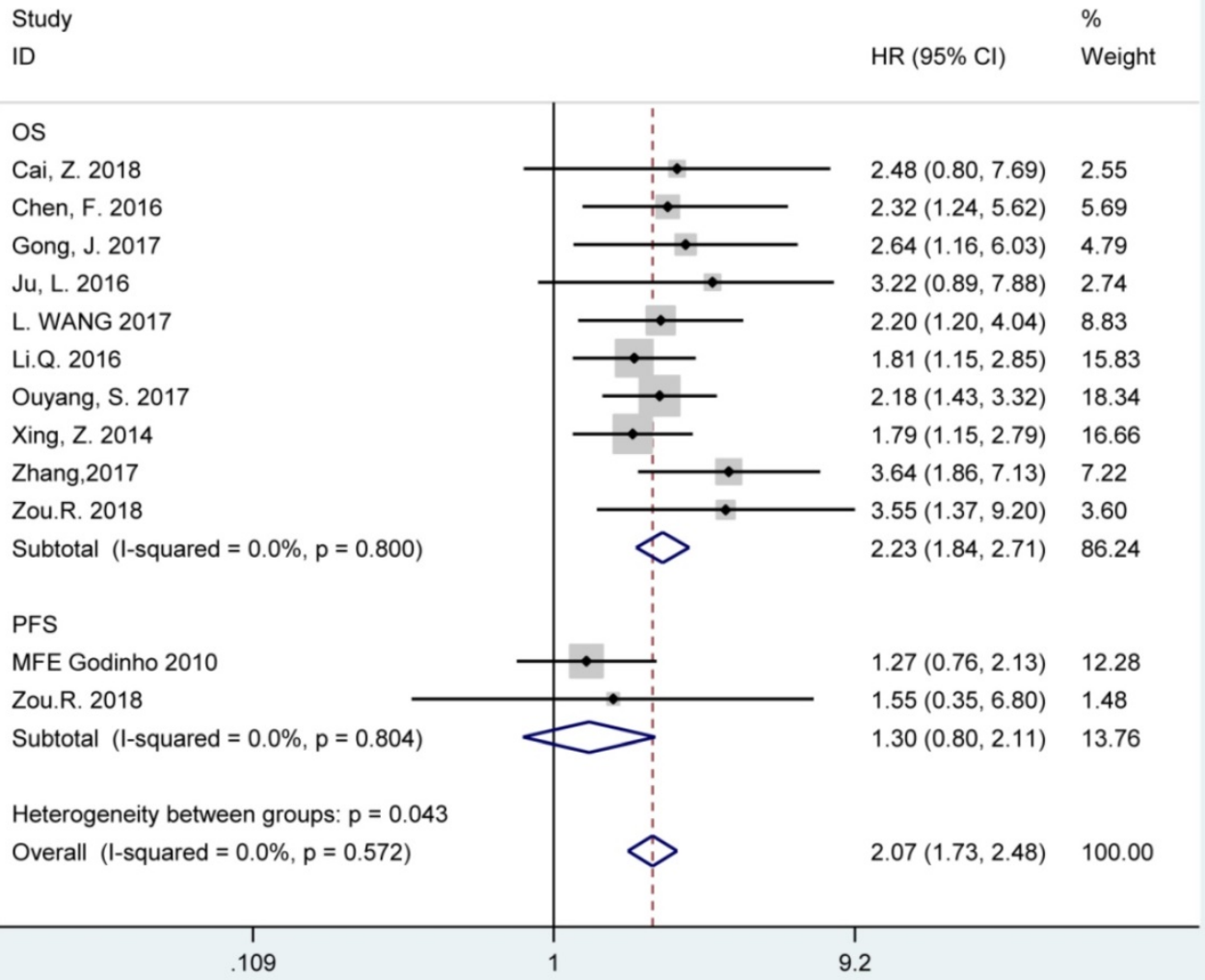

Figure 2. Forest plots for the association between BCAR4 expressions with overall survival (OS) and progression-free survival (PFS).

Table 2. Stratified analyses of the pooled HRs of overall survival with over-expressed BCAR4 in subgroup cancer patients.

\begin{tabular}{|c|c|c|c|c|c|}
\hline Subgroups & Studies & HR $(95 \%$ CI) & $\begin{array}{l}\text { Significance } \\
(P \text {-value })\end{array}$ & Model & $\begin{array}{l}\text { Heterogeneity } \\
I^{2}, P \text {-value }\end{array}$ \\
\hline \multicolumn{6}{|l|}{1 Tumor type } \\
\hline $1.1 \mathrm{BC}$ & 2 & $2.44(1.22,4.85)$ & 0.011 & Random & $66.6 \%, 0.084$ \\
\hline 1.2 GI cancer & 3 & $2.04(1.55,2.69)$ & 0.003 & Random & $0 \%, 0.810$ \\
\hline 1.3 Osteosarcoma & 2 & $2.58(1.38,4.80)$ & $<0.001$ & Random & $0 \%, 0.627$ \\
\hline 1.4 Others & 3 & $2.87(1.66,4.95)$ & $<0.001$ & Random & $0 \%, 0.863$ \\
\hline \multicolumn{6}{|l|}{2 Sample size } \\
\hline $2.1<100$ & 6 & $2.28(1.78,2.91)$ & $<0.001$ & Fixed & $0 \%, 0.693$ \\
\hline $2.2 \geq 100$ & 4 & $2.15(1.56,2.97)$ & $<0.001$ & Fixed & $0 \%, 0.521$ \\
\hline \multicolumn{6}{|c|}{3 Follow up (months) } \\
\hline $3.1 \leq 60$ & 6 & $2.42(1.82,3.22)$ & $<0.001$ & Fixed & $0 \%, 0.597$ \\
\hline $3.2>60$ & 4 & $2.08(1.60,2.71)$ & $<0.001$ & Fixed & $0 \%, 0.767$ \\
\hline \multicolumn{6}{|c|}{4 Survival analysis method } \\
\hline 4.1 Univariate & 5 & $2.10(1.67,2.65)$ & $<0.001$ & Fixed & $0 \%, 0.463$ \\
\hline 4.2 Multivariate & 5 & $2.57(1.80,3.66)$ & $<0.001$ & Fixed & $0 \%, 0.920$ \\
\hline
\end{tabular}

Abbreviations: BC, breast cancer; HR, hazard ratio; GI, gastrointestinal.

Table 3. Analysis of the pooled ORs of other clinicopathological features with over-expressed BCAR4 in cancer patients.

\begin{tabular}{|c|c|c|c|c|c|c|}
\hline Outcome & No. of Studies & No. of Participants & OR (95\% CI) & $P$ value & Model & $\begin{array}{l}\text { Heterogeneity } \\
\mathrm{Chi}^{2}, P \text {-value, } I^{2}\end{array}$ \\
\hline Age & 5 & 318 & $1.38(0.87,2.20)$ & 0.18 & Fixed & $5.27,0.26,24 \%$ \\
\hline Gender & 6 & 466 & $1.01(0.69,1.47)$ & 0.96 & Fixed & $2.01,0.85,0 \%$ \\
\hline Clinical stage & 7 & 610 & $3.28(2.33,4.60)$ & $<0.00001$ & Fixed & $4.21,0.66,0 \%$ \\
\hline Tumor size & 2 & 228 & $1.26(0.75,2.12)$ & 0.46 & Random & $3.62,0.06,72 \%$ \\
\hline LNM & 5 & 416 & $3.00(1.95,4.63)$ & $<0.00001$ & Fixed & $7.36,0.12,46 \%$ \\
\hline DM & 3 & 296 & $3.36(1.88,5.98)$ & $<0.0001$ & Fixed & $0.61,0.74,0 \%$ \\
\hline
\end{tabular}

Abbreviations: DM, distant metastasis; LNM, lymph node metastasis; OR, odds ratio. 
A

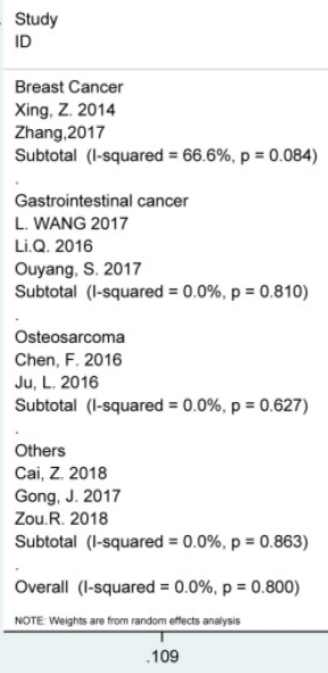

C

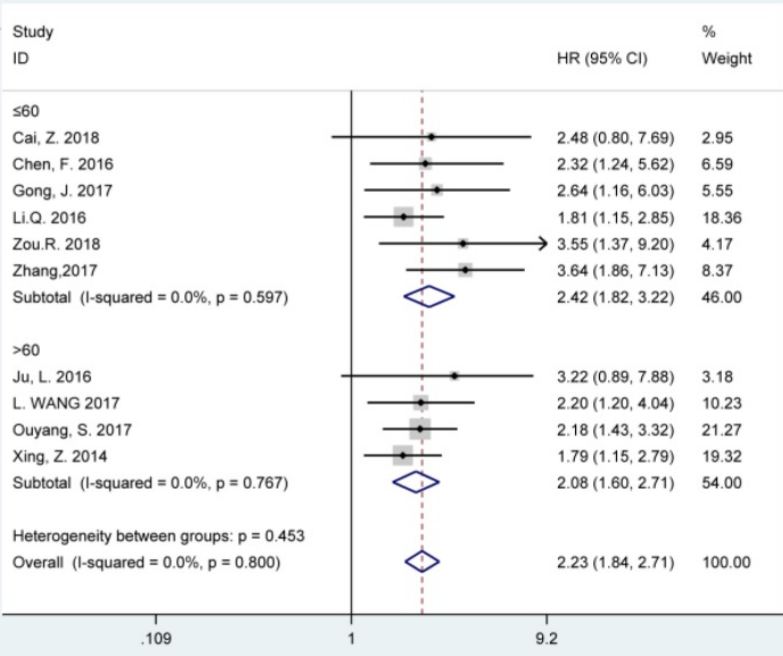

B

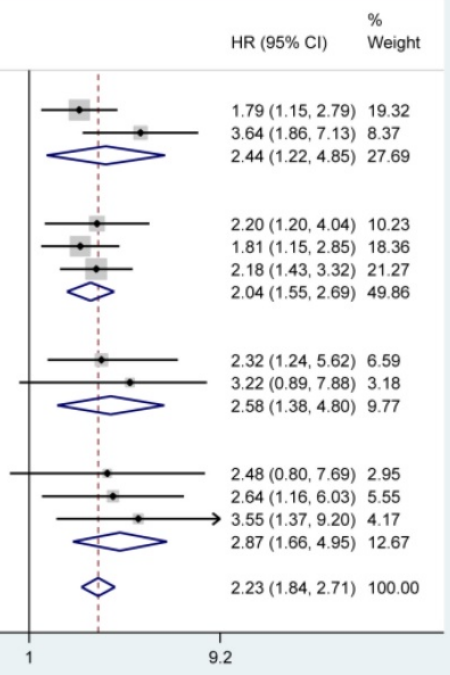

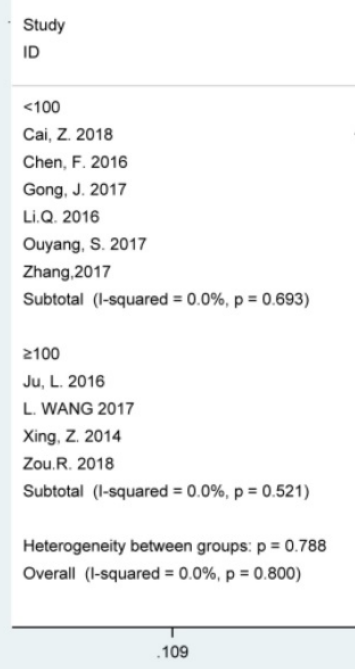

D

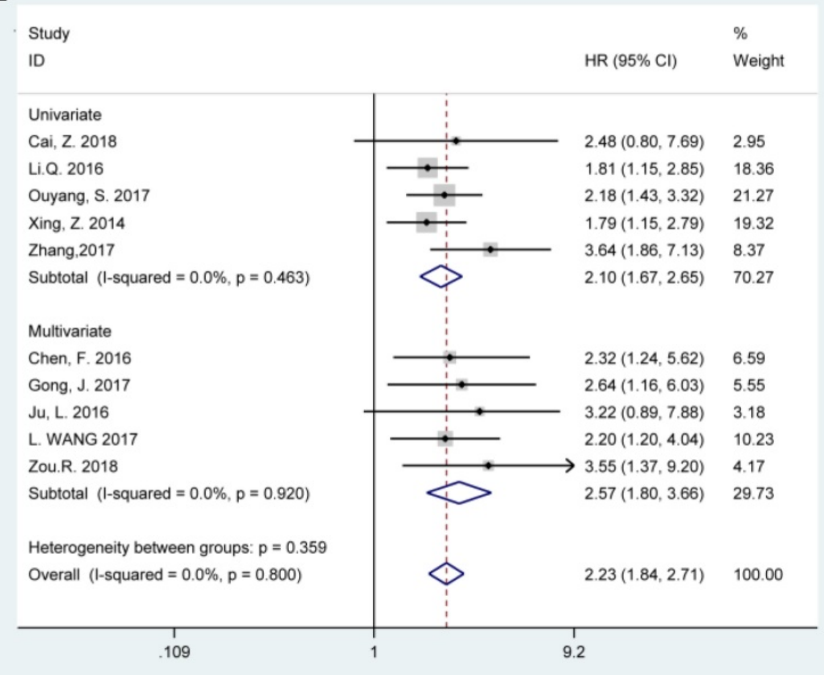

Figure 3. Stratified analyses for the correlation between BCAR4 expressions with overall survival (OS). Subgroup analysis of pooled HRs of OS by factor of tumor type (A), sample size (B), follow-up months (C), and survival analysis method (D) were presented respectively.

\section{Sensitivity analysis and publication bias}

The reliability of the crude results was evaluated by sensitivity analysis. After exclusion of any individual study, the combined effect of the pooled HR revealed no significant change, and therefore the results regarding BCAR4 expression for OS are considered to be credible (Figure 6).

The potential publication bias of the present meta-analysis was assessed by Begg's funnel plot, as well as Egger's test. However, the Egger's regression test indicated significant bias, and the shape of Begg's funnel plot also revealed evidence of asymmetry (Figure 7A). Therefore, we performed nonparametric "trim-and-fill" method by adding four missing studies as previously described (Figure 7B) [38]. The pooled HR and corresponding $95 \%$ CI for BCAR4 expression on OS was 2.01 (1.69-2.40) after adjustment.

\section{Different BCAR4 expression levels in cancer from TCGA and GTEx database}

In addition, we compared BCAR4 expression levels between cancerous and normal tissues in patients with different kinds of cancers using TCGA and GTEx datasets to verify the expression status or levels of lncRNA BCAR4. A majority of cancers showed higher expression of lncRNA BCAR4 in tumor tissues when compared with normal tissues, including sarcoma (SARC), breast invasive carcinoma (BRCA), cervical squamous cell carcinoma and endocervical adenocarcinoma (CESC), lung adenocarcinoma (LUAD), lung squamous cell carcinoma (LUSC), prostate adenocarcinoma (PRAD), and stomach adenocarcinoma (STAD). The details are showed in Figure S1. 


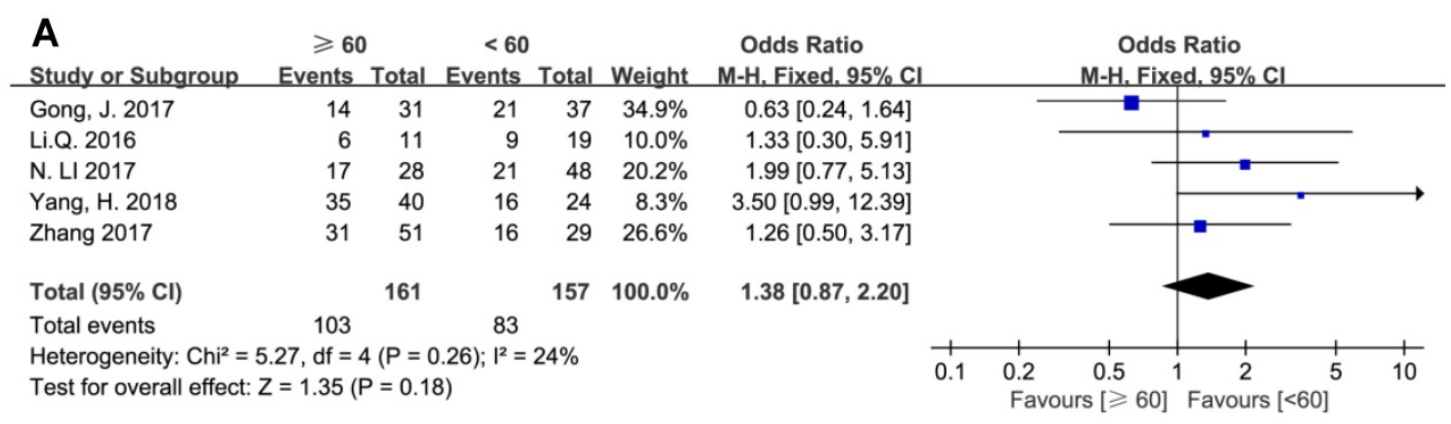

\begin{tabular}{|c|c|c|c|c|c|c|}
\hline $\begin{array}{l}\text { B } \\
\text { Study or Subqroup }\end{array}$ & $\begin{array}{c}\text { Male } \\
\text { Events }\end{array}$ & Total & $\begin{array}{l}\text { Female } \\
\text { Events }\end{array}$ & & Weight & $\begin{array}{l}\text { Odds Ratio } \\
\text { M-H. Fixed. } 95 \% \mathrm{Cl}\end{array}$ \\
\hline Chen, F. 2016 & 17 & 33 & 13 & 27 & $12.8 \%$ & $1.14[0.41,3.17]$ \\
\hline Gong, J. 2017 & 20 & 40 & 15 & 28 & $16.3 \%$ & $0.87[0.33,2.28]$ \\
\hline Ju, L. 2016 & 18 & 33 & 20 & 43 & $14.6 \%$ & $1.38[0.56,3.43]$ \\
\hline Li.Q. 2016 & 8 & 15 & 7 & 15 & $6.0 \%$ & $1.31[0.31,5.48]$ \\
\hline N. LI 2017 & 49 & 100 & 38 & 68 & $42.6 \%$ & $0.76[0.41,1.41]$ \\
\hline Yang, H. 2018 & 29 & 35 & 22 & 29 & $7.6 \%$ & $1.54[0.45,5.23]$ \\
\hline Total (95\% Cl) & & 256 & & 210 & $100.0 \%$ & $1.01[0.69,1.47]$ \\
\hline Total events & 141 & & 115 & & & \\
\hline \multicolumn{7}{|c|}{ Heterogeneity: $\mathrm{Ch}^{2}=2.01, \mathrm{df}=5(\mathrm{P}=0.85) ; \mathrm{l}^{2}=0 \%$} \\
\hline
\end{tabular}

Test for overall effect: $Z=0.05(P=0.96)$

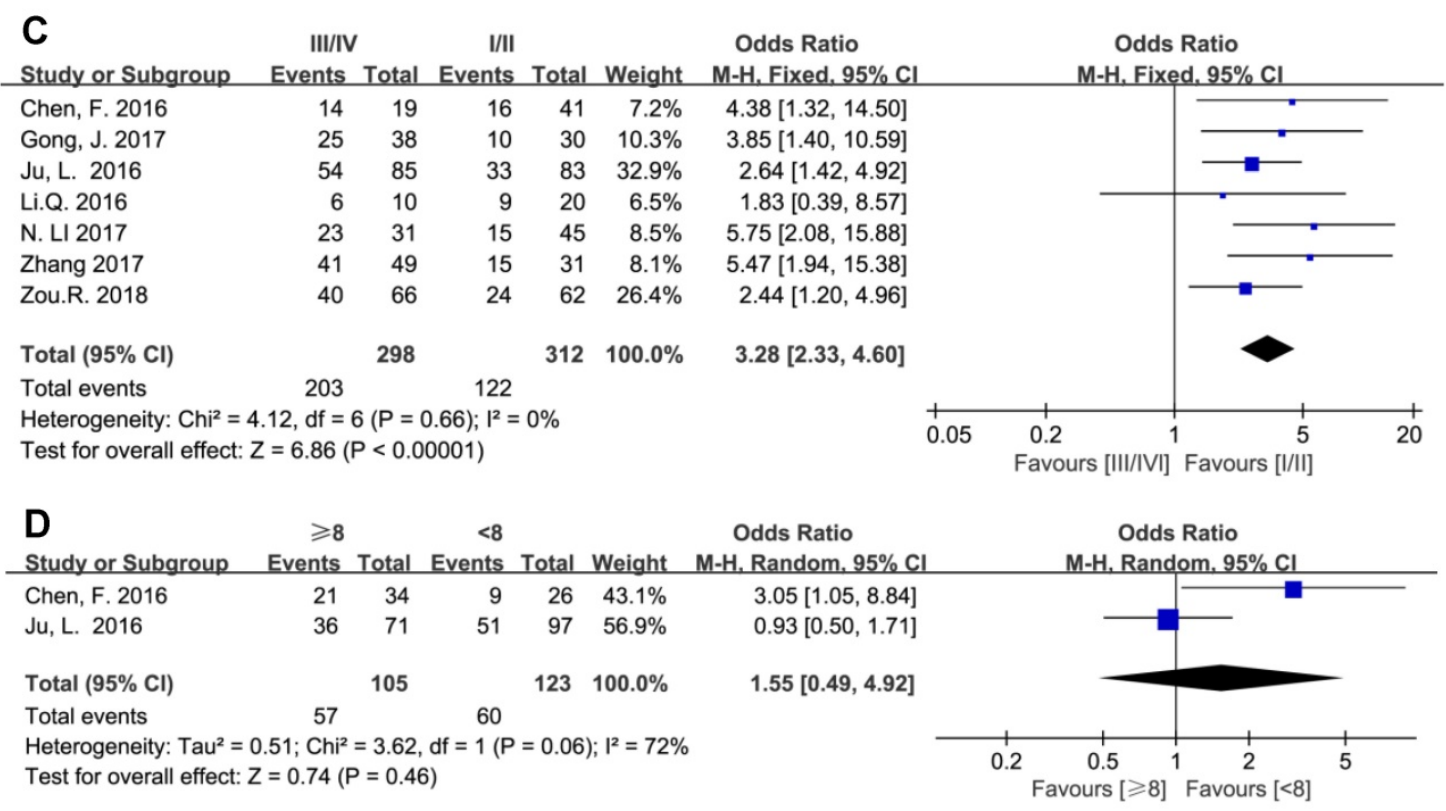

Figure 4. Forest plots for the association between BCAR4 expressions with other clinicalpathologic features, including age (A), gender (B), clinical stage (C) and tumor size (D).

\section{Discussions}

LncRNAs are non-protein-coding transcripts that were previously defined as chunk RNA and transcriptional "noise" [39]. However, this perception has been steadily replaced in the past few years since recent advancements in surveying mechanisms of lncRNAs have provided tools to functionally annotate these transcripts in diverse cellular processes [11, 40]. With rapid development of next-generation sequencing technique, mounting researches have uncovered the role of lncRNAs in regulating target gene expression as oncogenic or tumor suppressors
[41]. Consequently, lncRNAs have been proposed as promising biomarkers for early detection and accurate prognosis for various neoplasms nowadays [42-44].

Recent studies have investigated the association between lncRNA BCAR4 and human cancers, and the results show that high expression of BCAR4 indicates aggressiveness and poor prognosis in various carcinomas [19], including osteosarcoma [27, 28], breast cancer $[20,21,45,46]$, non-small cell lung cancer (NSCLC) [23, 25], gastric cancer [29], prostate cancer [26], colorectal cancer [31, 32, 47], and cervical cancer [30]. Moreover, higher expression levels of BCAR4 in most cancer tissues compared with normal 
tissues were verified using TCGA and GTEx databases. However, results from these studies should be interpreted with caution due to the limited sample size and discrete outcomes. A meta-analysis has been conducted by Zhao W. and colleagues [19] to demonstrate the pooled prognostic value of BCAR4 in human cancers. However, only nine studies were included without extensively searching other databases or providing information regarding BCAR4 and other clinical parameters, such as PFS, tumor size, gender and age. Of note, one study performed by MFE Godinho [20] does not contain data regarding the comparison of OS between high and low expression levels of BCAR4, whose inclusion in the meta-analysis might introduce possible bias to Zhao's results [19]. Therefore, we conducted this updated comprehensive meta-analysis to further investigate the prognostic role of BCAR4 in various cancers. Thirteen studies with seven cancer types containing 1,128 patients were pooled together in this study, and the results indicate that promoted BCAR4 expression was markedly associated with poor prognosis of OS, but not PFS in patients with a variety of cancers. Furthermore, subgroup stratified analysis showed that tumor type, sample size, and follow-up months, and survival analysis method did not alter the predictive value of BCAR4 on OS in human cancers. In addition, elevated BCAR4 level was markedly correlated with advanced (III/IV) clinical TNM stage, and dramatically predicted LNM and DM. However, the included single cohort studies showed that there were no significant difference between BCAR4 expression and TNM stage, LNM and DM, which were discordant with the pooled results due to the limited sample size $[27,31]$. Besides, the pooled OR implied BCAR4 expression levels were not associated with age, gender or tumor size. Despite a mild publication bias regarding BCAR4 expression for OS was observed in the study, the adjusted estimated value $(\mathrm{HR}=2.01,95 \% \quad \mathrm{CI}: 1.69-2.40)$ was not significantly different from the previous data $(\mathrm{HR}=2.23$, 95\% CI: 1.84-2.71) after using the "trim-and-fill" method, indicating the credibility of our results.

As one of the promising prognostic biomarkers with high accuracy for various patients, BCAR4 has also been claimed to be involved in diverse biological processes in cancers [48]. For instance, BCAR4 could activate mTOR pathway to induce cell proliferation and migration in chondrosarcoma [49], and regulate the expression of $\beta$-catenin by Wnt signaling pathway to promote the drug-resistance in gastric and breast cancer $[20,29]$. Besides, BCAR4 could wire up the Hippo pathway effector- Yes-associated protein (YAP) and Hedgehog (Hh) signaling to reprogramme glucose metabolism in breast cancer [18]. Co-expression of BCAR4 and low level of ERBB2 occurs frequently and indicate a worse PFS outcome for breast cancer patients undergoing tamoxifen resistance [45]. Moreover, the overexpression of BCAR4 could upregulate glioma-associated oncogene 2 (GLI2) level and promote cancer cell viability, migration and invasion both in vitro and in vivo [24].

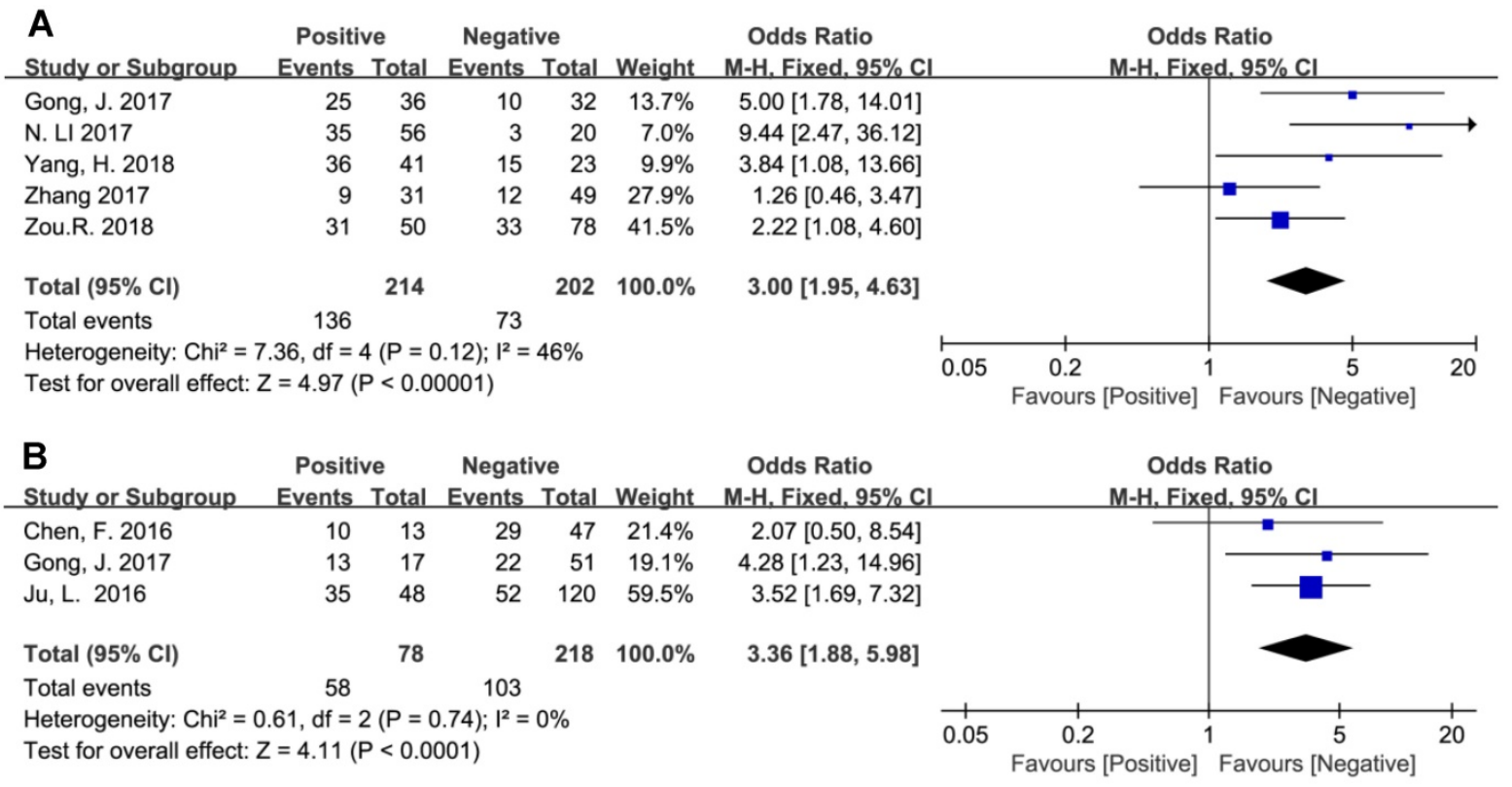

Figure 5. Forest plots for the association between BCAR4 expression with lymph node metastasis (A) and distant metastasis (B). 


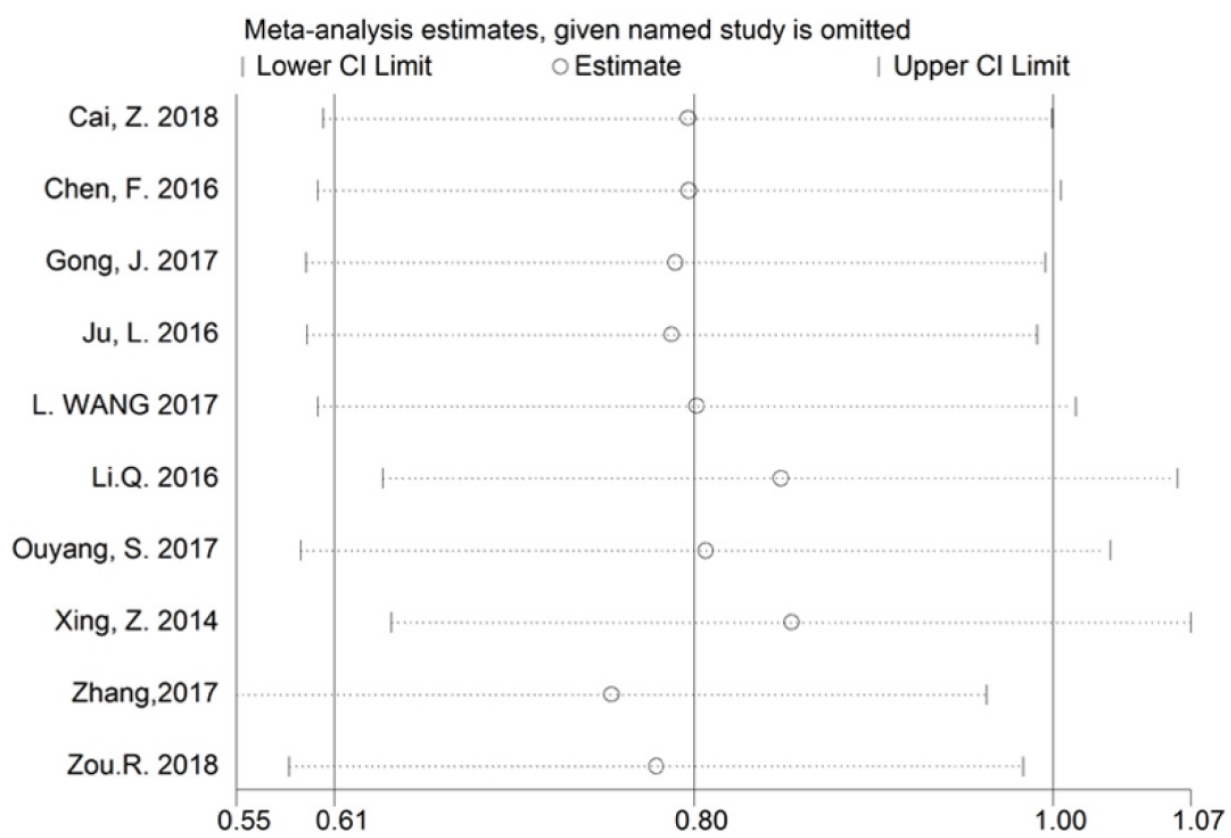

Figure 6. Sensitivity analysis of BCAR4 expression for overall survival (OS).
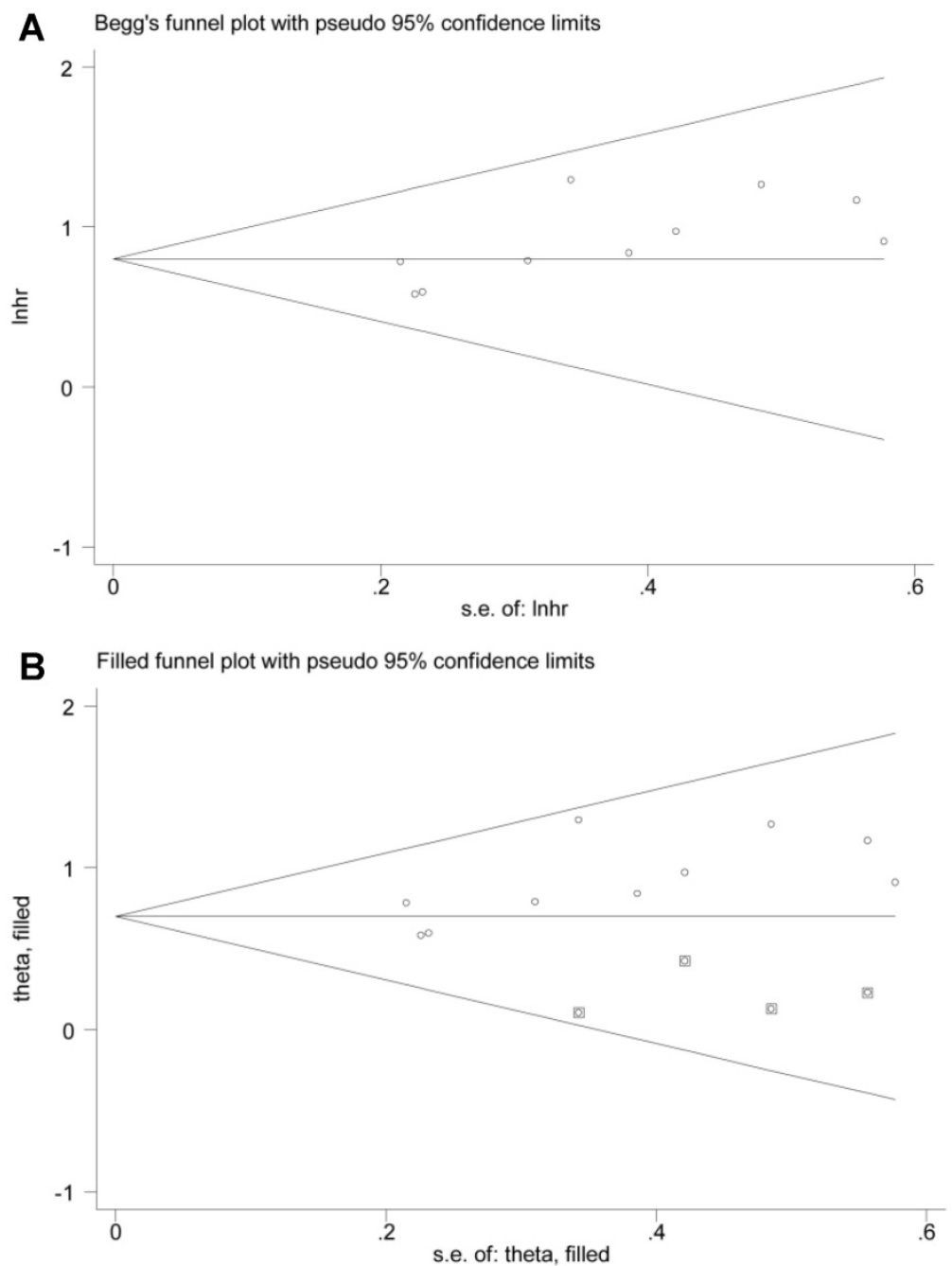

Figure 7. Publication bias of BCAR4 expression for overall survival (OS): Begg's funnel plot (A) and filled funnel plot (B) after adjustment by using the "trim-and-fill" method. 
Whereas BCAR4 knockdown could significantly suppress tumor cell proliferation, invasion and metastasis, as well as induce cell cycle arrest and increase apoptosis in NSCLC and cervical cancer [25, 50]. In addition, BCAR4 could mediate either canonical or non-canonical $\mathrm{Hh}$ cascade to activate GLI2-dependent gene transcription [30, 48], or regulate epithelial-mesenchymal transition (EMT) [49], and subsequently promote cell growth, metastasis and invasion in breast cancer and non-small cell lung cancer, or contribute to castration resistance in prostate cancer [26].

Taken together, the results of our comprehensive meta-analysis have demonstrated that BCAR4 expression is strong associated with unfavorable OS outcome and aggressive clinical features including metastasis and progression, suggesting an independent prognostic value for BCAR4 in human cancers and providing some insights for further research. However, it should be noted that several limitations still remain. First, some of the HRs were calculated by reconstructing survival curves rather than directly obtained from the original data, which might induce inevitable bias. Second, the cutoff value for BCAR4 expression varied across different studies due to the difficulty in reaching a consensus value, thus may introduce possible bias to the meta-analysis. Third, a majority of patients enrolled in our study were from China, except for one from the US and another from the Netherlands. Since discrepancy may exist among different races, our results may not be able to generalize to a larger spectrum of patients in other ethnicities and regions. Fourth, sample size of these studies is still small. Well-designed cohort studies with a larger sample size need to be carried out to further validate our results. Finally, the underlying mechanisms of BCAR4 in cancer progression still remain poorly understood, and thus more effort should be expanded to thoroughly elucidate the causative link between BCAR4 and human cancers.

In conclusion, the results of our study demonstrated strong correlation of BCAR4 with unfavorable survival outcome and clinical features including metastasis and progression, implicating an independent prognostic value for BCAR4 in human cancers. However, it should be noted that well-designed clinical studies with larger sample size are still warranted to clarify the predictive role of BCAR4 in cancer prognosis in future.

\section{Supplementary Material}

Supplementary figures.

http://www.jcancer.org/v10p5992s1.pdf

\section{Abbreviations}

BC: breast cancer; BCAR4: breast cancer anti-estrogen resistance 4; BRCA: breast invasive carcinoma; CI: confidence interval; CESC: cervical squamous cell carcinoma and endocervical adenocarcinoma; CNKI: China National Knowledge Infrastructure; CRC: colorectal cancer; DFS: disease-free survival; DM: distant metastasis; EMT: epithelial-mesenchymal transition; GC: gastric cancer; GEPIA: Gene Expression Profiling Interactive Analysis; GI: gastrointestinal; GLI2: glioma-associated oncogene 2; Hh: Hedgehog; HR: hazard ratio; K-M: Kaplan-Meier; lncRNA: Long non-coding RNA; LNM: lymph node metastasis; LUAD: lung adenocarcinoma; LUSC: lung squamous cell carcinoma; miRNAs: microRNAs; MFS: metastasis-free survival; N/A: not available; ncRNA: Non-coding RNA; NOS: Newcastle-Ottawa Scale; NSCLC: non-small cell lung cancer; OR: odds ratio; OS: overall survival; PC: Prostate cancer; PFS: progression-free survival; piRNA: piwi-interacting RNA; PRAD: prostate adenocarcinoma; RFS: recurrence-free survival; SARC: sarcoma; siRNA: short interfering RNA; STAD: stomach adenocarcinoma; TCGA: The Cancer Genome Atlas; YAP: Yes-associated protein.

\section{Acknowledgments}

This work was financially supported by the Natural Science Foundation of Hunan Province, China (2017DK2013, 2018JJ3716, and 2018JJ3759), China Scholarship Council (201806375067, 201806375068) and National Natural Science Foundation of China $(81902745,81372180)$.

\section{Authors' contributions}

CT and XLR contributed to the conception and design, data acquisition, analysis and interpretation. $\mathrm{JYH}, \mathrm{CHZ}$ and RQC participated in the design of the study, searched and selected the trials and assisted in the statistical analysis. WCW revised the manuscript. ZHL contributed to article drafting, critical revision and final approval of the version to be published. All authors agreed to be accountable for all aspects of the work.

\section{Competing Interests}

The authors have declared that no competing interest exists.

\section{References}

1. Bray F, Ferlay J, Soerjomataram I, et al. Global cancer statistics 2018 : GLOBOCAN estimates of incidence and mortality worldwide for 36 cancers in 185 countries. CA Cancer J Clin. 2018; 68(6): 394-424.

2. Sayles LC, Breese MR, Koehne AL, et al. Genome-Informed Targeted Therapy for Osteosarcoma. Cancer Discov. 2019; 9(1): 46-63. 
3. Wei SC, Duffy CR, Allison JP. Fundamental Mechanisms of Immune Checkpoint Blockade Therapy. Cancer Discov. 2018; 8(9): 1069-1086.

4. Zhang Y, Lun L, Li H, et al. The Value of IncRNA NEAT1 as a Prognostic Factor for Survival of Cancer Outcome: A Meta-Analysis. Sci Rep. 2017; 7(1): 13080.

5. Cai J, Zuo XL, Chen ZQ, et al. Long Noncoding RNAs Serve as Potential Diagnostic Biomarkers for Colorectal Cancer. J Cancer. 2019; 10(3): 611-619.

6. Su M, Xiao YH, Tang JM, et al. Role of lncRNA and EZH2 Interaction/Regulatory Network in Lung Cancer. J Cancer. 2018; 9(22): 4156-4165.

7. Zhang ZC, Tang $\mathrm{C}$, Dong $\mathrm{Y}$, et al. Targeting the long noncoding RNA MALAT1 blocks the pro-angiogenic effects of osteosarcoma and suppresses tumour growth. Int J Biol Sci. 2017; 13(11): 1398-1408.

8. Lee JT. Epigenetic regulation by long noncoding RNAs. Science. 2012; 338(6113): 1435-1439

9. Yang S, Sun Z, Zhou Q, et al. MicroRNAs, long noncoding RNAs, and circular RNAs: potential tumor biomarkers and targets for colorectal cancer. Cancer Manag Res. 2018; 10: 2249-2257.

10. Gao C, Lu W, Lou W, et al. Long noncoding RNA HOXC13-AS positively affects cell proliferation and invasion in nasopharyngeal carcinoma via modulating miR-383-3p/HMGA2 axis. J Cell Physiol. 2019; 234(8): 12809-12820.

11. Schmitt AM, Chang HY. Long Noncoding RNAs in Cancer Pathways. Cancer cell. 2016; 29(4): 452-463.

12. He J, Tu C, Liu Y. Role of lncRNAs in aging and age-related diseases. Aging Med. 2018; 1(2): 158-175.

13. Liu Y, Lin L, Zou R, et al. MSC-derived exosomes promote proliferation and inhibit apoptosis of chondrocytes via lncRNA-KLF3-AS1/miR-206/GIT1 axis in osteoarthritis. Cell cycle. 2018; 17(21-22): 2411-2422

14. Yu D, Tang C, Liu $P$, et al. Targeting lncRNAs for cardiovascular therapeutics in coronary artery disease. Curr Pharm Des. 2018; 24(1): 1-7.

15. Huang $\mathrm{W}$, Su G, Huang $X$, et al. Long noncoding RNA PCAT6 inhibits colon cancer cell apoptosis by regulating anti-apoptotic protein ARC expression via EZH2. Cell Cycle. 2019; 18(1): 69-83.

16. Shi Y, Li J, Liu Y, et al. The long noncoding RNA SPRY4-IT1 increases the proliferation of human breast cancer cells by upregulating ZNF703 expression. Mol Cancer. 2015; 14(1): 51.

17. Wu T, Du Y. LncRNAs: From Basic Research to Medical Application. Int J Biol Sci. 2017; 13(3): 295-307.

18. Zheng $X$, Han H, Liu GP, et al. LncRNA wires up Hippo and Hedgehog signaling to reprogramme glucose metabolism. EMBO J. 2017; 36(22): 3325-3335.

19. Zhao W, Wang Z, Fang $X$, et al. Long noncoding RNA Breast cancer antiestrogen resistance 4 is associated with cancer progression and its significant prognostic value. J Cell Physiol. 2019; 234(8):12956-12963.

20. Godinho MF, Sieuwerts AM, Look MP, et al. Relevance of BCAR4 in tamoxifen resistance and tumour aggressiveness of human breast cancer. Br J Cancer. 2010; 103(8): 1284-1291.

21. Xing $\mathrm{Z}$, Lin $\mathrm{A}, \mathrm{Li} \mathrm{C}$, et al. IncRNA directs cooperative epigenetic regulation downstream of chemokine signals. Cell. 2014; 159(5): 1110-1125.

22. Zhang JB, Song W, Wang YY, et al. MiR-133 manages effect of BCAR4 on migration and invasion of the breast cancer cells through Notch1 signaling pathway. Chin J Cancer Biother. 2017; 24(7): 733-741.

23. Gong J, Zhang H, He L, et al. Increased Expression of Long Non-Coding RNA BCAR4 Is Predictive of Poor Prognosis in Patients with Non-Small Cell Lung Cancer. Tohoku J Exp Med. 2017; 241(7): 29-34.

24. Yang H, Yan L, Sun K, et al. LncRNA BCAR4 increases viability, invasion and migration non-small cell lung cancer cells by targeting glioma-associated oncogene 2(GLI2). Oncol Res. 2019; 27(3): 359-369.

25. Li N, Gao WJ, Liu NS. LncRNA BCAR4 promotes proliferation, invasion and metastasis of non-small cell lung cancer cells by affecting epithelial-mesenchymal transition. Eur Rev Med Pharmacol Sci. 2017; 21(9): 2075-2086.

26. Cai Z, Wu Y, Li Y, et al. BCAR4 activates GLI2 signaling in prostate cancer to contribute to castration resistance. Aging (Albany NY). 2018; 10(12): 3702-3712.

27. Chen F, Mo J, Zhang L. Long noncoding RNA BCAR4 promotes osteosarcoma progression through activating GLI2-dependent gene transcription. Tumour Biol. 2016; 37(10): 13403-13412.

28. Ju L, Zhou YM, Yang GS. Up-regulation of long non-coding RNA BCAR4 predicts a poor prognosis in patients with osteosarcoma, and promotes cell invasion and metastasis. Eur Rev Med Pharmacol Sci. 2016; 20(21): 4445-4451.

29. Wang L, Chunyan $\mathrm{Q}$ Z Zhou $\mathrm{Y}$, et al. BCAR4 increase cisplatin resistance and predicted poor survival in gastric cancer patients. Eur Rev Med Pharmacol Sci. 2017; 21(18): 4064-4070.

30. Zou R, Chen $X$, Jin $X$, et al. Up-regulated BCAR4 contributes to proliferation and migration of cervical cancer cells. Surg Oncol. 2018; 27(2): 306-313.

31. Li Q, Dai Y, Wang F, et al. Differentially expressed long non-coding RNAs and the prognostic potential in colorectal cancer. Neoplasma. 2016; 63(6): 977-983.

32. Ouyang $S$, Zheng $X$, Zhou $X$, et al. LncRNA BCAR4 promotes colon cancer progression via activating $\mathrm{Wnt} /$ beta-catenin signaling. Oncotarget. 2017; 8(54): 92815-92826

33. Moher D, Liberati A, Tetzlaff J, et al. Preferred reporting items for systematic reviews and meta-analyses: the PRISMA statement. PLoS Med. 2009; 6(7): e1000097.
34. Moher D, Shamseer L, Clarke M, et al. Preferred reporting items for systematic review and meta-analysis protocols (PRISMA-P) 2015 statement. Syst Rev. 2015; 4(1): 1 .

35. Xu L, Yan N, Li Z, et al. A comparison of fulvestrant plus a targeted agent with fulvestrant alone in hormone receptor-positive advanced breast cancer that progressed on prior endocrine therapy: a meta-analysis. Onco Targets Ther. 2018; 11(12): 8389-8398.

36. Stang A. Critical evaluation of the Newcastle-Ottawa scale for the assessment of the quality of nonrandomized studies in meta-analyses. Eur J Epidemiol. 2010; 25(9): 603-605.

37. Tang Z, Kang B, Li C, et al. GEPIA2: an enhanced web server for large-scale expression profiling and interactive analysis. Nucleic Acids Res. 2019; 47(W1): W556-W560.

38. Weinhandl ED, Duval S. Generalization of trim and fill for application in meta-regression. Res Synth Methods. 2012; 3(1): 51-67.

39. Weidle UH, Birzele F, Kollmorgen G, et al. Long Non-coding RNAs and their Role in Metastasis. Cancer Genom Proteom. 2017; 14(3): 143-160.

40. Nagano T, Fraser P. No-nonsense functions for long noncoding RNAs. Cell. 2011; 145(2): 178-181.

41. Gao Q, Xie H, Zhan H, et al. Prognostic Values of Long Noncoding RNA GAS5 in Various Carcinomas: An Updated Systematic Review and Meta-Analysis. Front Physiol. 2017; 8: 814

42. Muller S, Raulefs S, Bruns P, et al. Next-generation sequencing reveals novel differentially regulated mRNAs, IncRNAs, miRNAs, sdRNAs and a piRNA in pancreatic cancer. Mol Cancer. 2015; 14(1): 94.

43. Murugan AK, Munirajan AK, Alzahrani AS. Long noncoding RNAs: emerging players in thyroid cancer pathogenesis. Endocr Relat Cancer. 2018; 25(2): R59-R82.

44. Li J, Cui Z, Li H, et al. Clinicopathological and prognostic significance of long noncoding RNA MALAT1 in human cancers: a review and meta-analysis. Cancer Cell Int. 2018; 18(1): 109.

45. Godinho MF, Wulfkuhle JD, Look MP, et al. BCAR4 induces antioestrogen resistance but sensitises breast cancer to lapatinib. Br J Cancer. 2012; 107(6): 947-955

46. van Agthoven $\mathrm{T}$, Dorssers LC, Lehmann U, et al. Breast Cancer Anti-Estrogen Resistance 4 (BCAR4) Drives Proliferation of IPH-926 lobular Carcinoma Cells. PloS one. 2015; 10(8): e0136845.

47. Dong L, Lin W, Qi P, et al. Circulating Long RNAs in Serum Extracellular Vesicles: Their Characterization and Potential Application as Biomarkers for Diagnosis of Colorectal Cancer. Cancer Epidemiol Biomarkers Prev. 2016; 25(7): 1158-1166.

48. Xing Z, Park PK, Lin C, et al. LncRNA BCAR4 wires up signaling transduction in breast cancer. RNA Biol. 2015; 12(7): 681-689.

49. Shui $\mathrm{XL}$, Zhou $\mathrm{CW}$, Lin $\mathrm{W}$, et al. Long non-coding RNA BCAR4 promotes chondrosarcoma cell proliferation and migration through activation of mTOR signaling pathway. Exp Biol Med. 2017; 242(10): 1044-1050.

50. Li Z, Shen J, Chan MT, et al. TUG1: a pivotal oncogenic long non-coding RNA of human cancers. Cell Prolif. 2016; 49(4): 471-475. 